\title{
FRECUENCIA E INTENSIDAD DE LOS MIEDOS EN LOS NIÑOS: DATOS NORMATIVOS
}

\author{
BONIFACIO SANDÍN, PALOMA CHOROT, ROSA M. VALIENTE \\ Y MIGUEL A. SANTED
}

Universidad Nacional de Educación a Distancia (UNED), Madrid

(Recibido el 10 de julio de 1997)

\begin{abstract}
En el presente estudio referimos los resultados de una investigación normativa sobre el miedo, basada en una muestra de niños normales españoles (con un rango de edad entre 9 y 11 años). Estudiamos la frecuencia, intensidad y contenido de los miedos utilizando la versión española del Fear Survey Schedule for Children-Revised (FSSC-R; Ollendick, 1983). Los patrones de miedos declarados por los niños españoles resultaron ser bastante semejantes a los obtenidos repetidamente con muestras de niños de Estados Unidos, Australia y Gran Bretaña, y no muy diferentes de los indicados para muestras de niños chinos. Las niñas informaron significativamente mayores niveles de temor (tanto en frecuencia como en intensidad) que los niños, y ambos (niños y niñas) informaron poseer altos niveles, tanto en la intensidad como en el número de miedos, en la dimensión de temores a peligros físicos y muerte. Algunas diferencias entre niños y niñas resultaron significativas respecto el contenido de los miedos, siendo los miedos a los pequeños animales los que, en este sentido, resultaron ser más discriminativos. Los resultados, así mismo, proporcionan un importante apoyo empírico para la validación de la versión española del FSSC-R.
\end{abstract}

Palabras clave: FSSC-R, miedos, prevalencia, intensidad, contenido, niños.

\section{Frequency and Intensity of fears in children: Normative data}

In the present study we report the findings of a normative fear investigation with a sample of Spanish normal children (ranging in age from 9 to 11 years). We examined the frequency, intensity and content of fear using the Spanish Fear Survey Schedule for Children-Revised (FSSC-R; Ollendick, 1983). The fears reported by the Spanish children were found to be quite similar to those found in American, Australian and British children, and not very different from fears reported by Chinese children. Girls reported significantly higher levels of fearfulness (frequency and intensity) than boys, and both self-reported high levels of intensity and number of fears on the physical danger and death domain. Some significant differences between girls and boys were found on the content of children fears, being small animal fears which best discriminated between girls and boys. Results provide also support for validation of the Spanish version of the FSSC-R.

Key words: FSSC-R, fears, prevalence, intensity, content, children.

\section{INTRODUCCIÓN}

El miedo constituye, por una parte, un componente necesario y unido inextricablemente al desarrollo evolutivo del individuo $\mathrm{y}$, por otra, puede ser el aspecto más insoportable y perturbador de la vida de una persona (Sandín, 1997, p.

Correspondencia: Bonifacio Sandín, Facultad de Psicologia, Universidad Nacional de Educación a Distancia, Apartado 60148, 28040 Madrid.
21). Definido como una reacción normal y adaptativa ante una amenaza real o imaginaria, el miedo constituye una parte integrada al desarrollo y maduración del ser humano. En este sentido, el miedo durante la infancia no sólo sirve para proteger al niño de peligros inminentes; también se ha sugerido que puede cumplir un papel relevante como factor favorecedor del vínculo entre el niño y las personas que lo cuidan (e.g., los padres). 
Los miedos no son fenómenos arbitrarios, ya que no todos los estímulos poseen la misma potencialidad de asociarse a las respuestas de miedo. La selectividad ("preparedness») de los miedos y las fobias ha sido sugerida, así mismo, como un fenómeno particularmente adaptativo y de gran valor y significado filogenético (para una revisión sobre este aspecto véase Sandín, 1995). Aunque los miedos pueden ser descritos, pues, como fenómenos normales, su investigación es claramente necesaria por su relevancia clínica. Es decir, por una parte se hace necesario establecer las normas que puedan servir como pauta general para diferenciar los miedos «normales» de las fobias (miedos clínicamente significativos) y, por otra, es preciso determinar con mayor precisión qué fases evolutivas se asocian más específicamente a diferentes tipos de miedos. El estudio de los miedos infantiles resulta también particularmente importante porque éstos pueden constituir la base de las fobias y otros trastornos de ansiedad y depresivos que aparecen en fases posteriores del desarrollo.

Sobre la base del empleo de procedimientos psicométricos de evaluación (cuestionarios de autoinforme), durante los últimos diez años se han producido importantes estudios normativos sobre los miedos en los niños y adolescentes (e.g., Dong, Yang y Ollendick, 1994; Gullone y King, 1992, 1993; McCathie y Spence, 1991; Ollendick, 1983; Ollendick, King y Frary, 1989; Ollendick, Matson y Hensel, 1985; Ollendick, Yule y Ollier, 1991; Pelechano, 1981, 1984; para una revisión véase Sandín, 1997). Estos estudios tienden a indicar que los miedos son fenómenos relativamente comunes durante la infancia y la adolescencia, que los temores poseen una tendencia evolutiva (suelen variar durante el curso del desarrollo), y que las niñas suelen referir más miedos (tanto en frecuencia como en intensidad) que los niños. A pesar de la existencia de patrones evolutivos en la expresión y desarrollo del miedo, la mayor parte de estos estudios normativos han constatado que los miedos más comunes, tanto en niños como en adolescentes, parecen referirse a temores vinculados a los peligros físicos y la muerte, tales como no poder respirar, ser atropellado por un coche, o caer desde lugares altos. Este fenómeno, aunque con ligeras diferencias, parece darse tanto en los niños como en las niñas.

Tales estudios se han llevado a cabo básicamente con el conocido cuestionario Fear Survey Schedule for Children-Revised (FSSC-R)(Ollendick, 1983), una forma revisada del FSSC de Scherer y Nakamura (1968). El FSSC-R se ha empleado profusamente no sólo en Estados Unidos de América, sino también en muchos otros países del mundo (e.g., Dong et al., 1994; King, Gullone y Ollendick, 1990; Ollendick et al., 1985, 1989, 1991). Estudios factoriales con el FSSC-R han constatado la existencia de 5 dimensiones básicas de miedos, referidas, respectivamente, a temores de tipo interpersonal-evaluativo, fenómenos desconocidos, miedos hacia los animales y daños menores, peligros mayores y muerte, y miedos de tipo médico (Ollendick, 1983; Ollendick et al., 1989). Muchos de estos estudios, que han evaluado las propiedades psicométricas de fiabilidad y validez del cuestionario han constatado, así mismo, que éstas son excelentes. El empleo del FSSC-R ha resultado particularmente útil como instrumento normativo para seleccionar niños con elevados niveles de miedos, y como instrumento clínico para evaluar las variaciones pre-/post-tratamiento.

La utilización del FSSC-R en poblaciones de habla española no sólo es útil sino incluso necesaria, al menos si deseamos establecer comparaciones con el grueso de los resultados sugeridos internacionalmente sobre los miedos de la infancia 
y adolescencia. Esta idea nos llevó a emplear el FSSC-R como instrumento de autoinforme de elección en nuestras investigaciones sobre el miedo infantojuvenil. Nuestros datos sugieren que la estructura factorial de los miedos, evaluada a través del FSSC-R, es equivalente a la postulada por el grupo de Ollendick, confirmando indirectamente su invarianza factorial transcultural de 5 dimensiones.

Mediante el presente estudio pretendemos presentar datos normativos preliminares sobre los miedos infantiles utilizando nuestra versión española del FSSC-R. Intentamos obtener información relativa a la prevalencia, intensidad y contenido de los miedos infantiles, haciendo especial énfasis en las posibles diferencias asociadas al sexo.

\section{MÉTODO}

\section{Participantes}

Todos los participantes pertenecían a la muestra general que empleamos en nuestro anterior estudio sobre los miedos infantiles (Sandín y Chorot, en prensa). La muestra para el presente estudio constaba de 151 participantes normales (no clínicos ni con retraso mental), la cual estaba constituida por 75 niños y 76 niñas, con edades que oscilaban entre 9 y 11 años. Todos poseían un nivel socioeconómico medio o medio-bajo. Los participantes asistían regularmente a colegios de enseñanza primaria y fueron seleccionados al azar en una zona urbana de la población escolar de la comunidad de Extremadura (Badajoz).

\section{Instrumentos y procedimiento}

Los participantes fueron evaluados en las propias aulas del centro docente don- de asistían con regularidad. Se les aplicó de forma colectiva la versión española del Fear Survey Schedule for ChildrenRevised (FSSC-R; Ollendick, 1983) llevada a cabo por Chorot y Sandín (Sandín, 1997) ${ }^{1}$. La versión española del FSSC-R, al igual que la versión en lengua inglesa, consta de 80 items. No obstante, el Item 73 , que en la versión original se refiere al miedo a Rusia, ha sido eliminado (por considerarlo irrelevante). A su vez, el Item 62 , que en la versión original se refiere al miedo a estar solo, se ha dividido en dos items, uno referido al miedo a estar solo en casa, y otro referido al miedo a estar solo fuera de casa. Datos psicométricos favorables sobre fiabilidad y validez factorial del FSSC-R han sido obtenidos recientemente por nosotros (Sandín y Chorot, 1998).

$\mathrm{Al}$ igual que en la versión original del cuestionario, cada item debía cumplimentarse de acuerdo con tres alternativas de respuesta, esto es, "Nada (1)», «Un poco (2)», y «Mucho(3) «, de acuerdo con el nivel de miedo que cada objeto o situación (representado en los respectivos items) solía producir en el sujeto. La puntuación total del cuestionario se computó sumando los valores obtenidos en cada item $(1,2$ o 3$)$, la cual puede oscilar entre 80 (mínima) y 240 (máxima). También se obtuvieron puntuaciones para cada una de las cinco subescalas del cuestionario.

Las subescalas se construyeron partiendo de los resultados de nuestro estudio factorial con el FSSC-R (Sandín y Chorot, 1998). Dichas subescalas se establecieron de la forma que sigue: (1) Miedos al fracaso y la crítica (miedos sociales) (17 items; e.g., "Que tus padres te regañen o critiquen", "Obtener malas notas»), (2) Miedos a pequeños animales y daños menores (13 items; e.g., «Las hor-

\footnotetext{
${ }^{1}$ La versión española del FSSC-R puede encontrarla el lector en: B. Sandín, Ansiedad, miedos y fobias en niños y adolescentes, Madrid, Dykinson, 1997.
} 
migas o los escarabajos», «Las lagartijas»), (3) Miedos a los peligros físicos y muerte (16 items; e.g., «Ser atropellado por un coche o un camión», "No poder respirar»), (4) Miedos a lo desconocido (17 items; e.g., «Estar en un lugar donde hay mucha gente», "Los lugares oscuros»), y (5) Miedos médicos (5 items; e.g., "Que te pongan una inyección», "Tener que ir al hospital») (véase Sandín y Chorot, 1998). Estas subescalas, aunque en diferente orden, coinciden conceptualmente con las cinco dimensiones referidas internacionalmente para el FSSC-R (e.g., Ollendick et al., 1989). Las puntuaciones de las subescalas se obtuvieron sumando el valor de todos los items que la componen. De este modo, las puntuaciones máxima y mínima posibles son como sigue: 17-51 (Miedos al fracaso y la crítica), 13-39 (Miedos a pequeños animales y daños menores), 16-48 (Miedos a peligros físicos y muerte), 17-51 (Miedos a lo desconocido), y 5-15 (Miedos médicos).

Antes de aplicarse el cuestionario, el evaluador (una estudiante de psicología de quinto curso) leyó las instrucciones con sumo cuidado, enfatizando cada una de las tres opciones de respuesta del mismo. Además, durante la realización de la prueba, el evaluador permaneció en el aula para asistir a los niños y atender cualquier duda relacionada con la contestación al cuestionario.

\section{RESULTADOS}

A continuación exponemos de forma separada los resultados relacionados con las diferentes medidas del miedo calculadas, esto es, la prevalencia (frecuencia), la intensidad, y el contenido. La prevalencia se ha obtenido, de acuerdo con los estudios previos basados en el FSSC-R, sumando los items estimados por el participante como generadores de mucho miedo (esto es, los items estimados con valor de 3 -miedos excesivos). Mediante esta medida se obtiene una indicación sobre el número de miedos excesivos. También hemos analizado los 10 miedos más comunes (i.e., los 10 miedos estimados con mayor frecuencia como temores excesivos por los participantes). La intensidad del miedo se cuantificó sumando las puntuaciones obtenidas en cada uno de los items, bien para la escala total (puntuación general), o bien para cada una de las subescalas por separado. Finalmente, analizamos las diferencias sexuales de acuerdo específicamente con el contenido de los miedos.

\section{Prevalencia de los miedos}

En la Tabla 1 indicamos el número total de miedos estimados por los participantes como temores excesivos (i.e., miedos estimados con valor 3 ). Como puede apreciarse, la muestra total refiere experimentar, como media, cerca de 20 miedos excesivos, lo cual denota un nivel de miedos notablemente elevado, y sólo ligeramente superior a los resultados indicados por el grupo de Ollendick (e.g. Dong et al., 1994; Ollendick et al., 1989). También puede observarse que los participantes parecen experimentar mayor número de miedos de la subescala relacionada con los miedos a la posibilidad de sufrir daños mayores (i.e., miedos al peligro y muerte).

Con objeto de analizar las posibles diferencias en la prevalencia de los miedos entre los niños y las niñas, se calculó un análisis de varianza multivariado (MANOVA) para la puntuación total en el FSSC-R y para las puntuaciones de las 5 subescalas, actuando la variable sexo como variable independiente (niños vs. niñas). El análisis resultó ser estadísticamente significativo [Hotelling $F(6,144)=$ $9,1, P<0,001$ ], lo cual sugería que las diferencias entre los niños y las niñas 
eran estadísticamente significativas. Posteriormente, y para contrastar las diferencias en cada una de las seis variables, se calcularon por separado 6 pruebas de $F$ univariadas (una para cada variable dependiente) (véase la Tabla 1). Como puede apreciarse, en todos los casos las niñas tienden a experimentar significativamente mayor número de miedos excesivos que los niños. No obstante, para las diferencias en los miedos de tipo social y miedos a los fenómenos desconocidos se evidencia un grado notablemente menor de significación estadística.

\section{Los 10 miedos más comunes}

Analizamos también los 10 miedos más prevalentes en nuestra muestra. Para ello calculamos los miedos más comunes estimados por los participantes con la máxima intensidad (con valor de 3). En la Tabla 2 referimos los diez miedos más comunes, especificando los valores separadamente para la muestra total y para las submuestras de niñas y niños respectivamente (la tabla incluye los 11 miedos más comunes en la muestra total, con objeto de poder reflejar los diez más frecuentes en ambos subgrupos; además, los tres últimos items referidos poseen idéntica frecuencia-i.e., 62,9\%). Diez de los 11 temores representados en la tabla pertenecen a la dimensión o subescala de miedos al peligro físico y muerte (el Item 15 - miedo a «ser enviado al director del colegio»-que es el séptimo miedo más común en la muestra total, forma parte de la dimensión de miedos socia-

Tabla 1. Prevalencia de los miedos (general y en las 5 subescalas) según sexo

\begin{tabular}{|c|c|c|c|c|c|c|c|}
\hline \multirow[b]{3}{*}{ Variable } & & & \multicolumn{4}{|c|}{ Sexo } & \\
\hline & \multicolumn{2}{|c|}{ Total } & \multicolumn{2}{|c|}{ Niños } & \multicolumn{2}{|c|}{ Niñas } & \multirow[b]{2}{*}{$F(1,149)$} \\
\hline & Media & DT & Media & DT & Media & DT & \\
\hline FSSC-R General & 19,8 & 10,1 & 15,1 & $\mathbf{9 , 8}$ & 24,6 & 9,7 & $33,1^{\cdots}$ \\
\hline Miedos al fracaso y la crítica & 4,0 & 3,4 & 3,2 & 3,1 & 4,8 & 3,4 & $10,1^{\prime}$ \\
\hline Miedos a pequeños animales & 2,4 & 2,2 & 1,4 & 1,5 & 3,4 & 2,5 & $40,6^{n \cdots}$ \\
\hline Miedos al peligro y muerte & 9,1 & 4,0 & 7,8 & 3,8 & 10,7 & 3,3 & $31,0 \ldots$ \\
\hline Miedos a lo desconocido & 2,8 & 2,5 & 2,0 & 2,4 & 3,4 & 2,7 & $12,7^{* *}$ \\
\hline Miedos médicos & 1,0 & 1,0 & 0,7 & 0,8 & 1,3 & 0,9 & $14,3^{\cdots \cdots}$ \\
\hline
\end{tabular}

Nota: Las pruebas de $F$ se refieren a las diferencias entre niños y niñas.

${ }^{\mathrm{P}} \mathrm{P}<0,05,{ }^{\circ} \mathrm{P}<0,01,{ }^{*} \mathrm{P}<0,001$ (se aplicó la corrección de Bonferroni).

Tabla 2. Los diez miedos más comunes (porcentaje de niños/as que los experimentan)

\begin{tabular}{lcll}
\hline Item FSSC-R & $\begin{array}{c}\text { Total } \\
(n=151)\end{array}$ & $\begin{array}{c}\text { Niños } \\
(n=75)\end{array}$ & $\begin{array}{c}\text { Niñas } \\
(n=76)\end{array}$ \\
\hline 41. Ser atropellado por un coche o camión & 87,3 & 81,3 & 93,3 \\
20. Los bombardeos (que tu país sea invadido) & 80,6 & 72,0 & 89,3 \\
76. No poder respirar & 74,0 & $66,2\left(4^{\circ}\right)$ & 81,6 \\
59. Recibir una descarga eléctrica & 70,7 & $69,3\left(3^{0}\right)$ & $72,0\left(11^{\circ}\right)$ \\
9. La muerte o la gente muerta & 67,5 & 57,3 & 77,6 \\
58. Caerse dese lugares altos & 65,6 & $54,7\left(7^{\circ}\right)$ & 76,3 \\
15. Ser enviado al director del colegio & 64,9 & $56,0\left(6^{0}\right)$ & $73,7\left(9^{\circ}\right)$ \\
34. El fuego o quemarse & 64,6 & 53,3 & 76,0 \\
26. Que un ladrón entre en tu casa & 62,9 & $44,0\left(12^{0}\right)$ & $81,6\left(4^{\circ}\right)$ \\
72. Los terremotos & 62,9 & 49,3 & $76,3\left(7^{\circ}\right)$ \\
70. Los microbios o padecer una enfermedad grave & 62,9 & $53,3\left(9^{\circ}\right)$ & $72,4\left(10^{\circ}\right)$ \\
\hline
\end{tabular}

Nota. Entre paréntesis se indica el lugar de orden que ocupa el item para el grupo especifico. 
les o miedos al fracaso y la crítica) (véase Sandín y Chorot, 1998). Son dignos de mención los elevados porcentajes de sujetos que parecen experimentar dichos miedos.

Podemos observar, así mismo, que, aun siendo particularmente elevados en ambos casos, los porcentajes son siempre superiores en el grupo de niñas que en el de niños. Refiriéndonos en concreto a las niñas, más del $80 \%$ dicen sentir miedo intenso a cuatro de los temores representados en la tabla, y más del $70 \%$ lo señalan con respecto a los restantes items. Por otra parte, aun cuando es muy semejante el orden de los miedos más comunes en los niños y en las niñas, observamos algunas diferencias. En primer lugar, el temor a sufrir una descarga eléctrica (Item 59), que en la muestra total y en la de niños ocupa el cuarto lugar, parece ser un temor relativamente menos frecuente entre las niñas, ya que en éstas ocupa el lugar décimo primero; téngase en cuenta, sin embargo, que en términos absolutos este miedo también es más prevalente en las niñas $(72,0 \%)$ que en los niños $(69,3 \%)$. Una segunda diferencia parece asociarse al Item 26 (temor a que un ladrón entre en casa), el cual no aparece entre los 10 miedos más comunes en el grupo de niños, mientras que resulta ser el cuarto temor más frecuente entre las niñas.

\section{Intensidad de los miedos}

Las medias y desviaciones típicas en intensidad de miedo, para las seis variables (escala general y 5 subescalas del FSSC-R), están referidas en la Tabla 3 , con indicación separada de los valores correspondientes a la muestra total, a los niños y a las niñas. Entre las 5 subescalas se observa que las medias más elevadas corresponden a la dimensión de miedos a peligros mayores (Miedos a peligros físicos y muerte). Además, las niñas parecían obtener puntuaciones siempre más elevadas que los niños. Puesto que pretendíamos estudiar las diferencias en la intensidad del miedo entre los grupos de niñas y niños, calculamos inicialmente un MANOVA, con una variable independiente (sexo) y 6 variables dependientes (FSSC-R general y las 5 subescalas). El MANOVA resultó ser estadísticamente significativo, Hotelling $F(6,144)=10,8$, $p<0,001$, sugiriéndose con ello diferencias significativas entre niños y niñas. La aplicación de análisis de varianza (ANOVA) univariados para cada una de estas seis variables (variable independiente $=$ sexo) demostró la existencia de diferencias significativas entre niños y niñas en todas ellas (véase la Tabla 3). La significación estadística resultó ser notablemente menor para la subescala de temores sociales (algo semejante a lo que

Tabla 3. Intensidad de los miedos (general y en las 5 subescalas) según sexo

\begin{tabular}{|c|c|c|c|c|c|c|c|}
\hline \multirow[b]{3}{*}{ Variable } & & & \multicolumn{5}{|c|}{ Sexo } \\
\hline & \multicolumn{2}{|c|}{ Total } & \multicolumn{2}{|c|}{ Niños } & \multicolumn{2}{|c|}{ Niñas } & \multirow[b]{2}{*}{$F(1,149)$} \\
\hline & Media & DT & Media & DT & Media & DT & \\
\hline FSSC-R General & 155,1 & 24,9 & 132,9 & 23,2 & 156,9 & 20,5 & $33,2^{\cdots \cdots}$ \\
\hline Miedos al fracaso y la crítica & 32,2 & 6,9 & 30,3 & 6,8 & 34,3 & 6,7 & $8,2^{*}$ \\
\hline Miedos a pequeños animales & 22,0 & 5,5 & 18,9 & 4,3 & 25,4 & 4,8 & $62,3^{* * *}$ \\
\hline Miedos al peligro y muerte & 38,8 & 6,2 & 36,1 & 6,2 & 41,7 & 4,6 & $25,6^{* *}$ \\
\hline Miedos a lo desconocido & 29,0 & 6,8 & 26,4 & 6,3 & 31,7 & 6,2 & $20,6 \ldots$ \\
\hline Miedos médicos & 8,9 & 2,4 & 8,2 & 2,3 & 9,7 & 2,3 & $15,8^{* * *}$ \\
\hline
\end{tabular}

Nota: Las pruebas de $F$ se refieren a las diferencias entre niños y niñas.

$" p<0,05, " p<0,01, \cdots p<0,001$ (se aplicó la corrección de Bonferroni). 
observamos en nuestros análisis sobre la prevalencia).

\section{Diferencias sexuales en el contenido de los miedos}

Para poder determinar qué tipos de miedos diferenciaban mejor a los niños de las niñas, llevamos a cabo análisis de regresión logística múltiple, actuando como variables independientes la medidas de miedos y como variable dependiente el sexo. Se utilizó el método de selección por pasos sucesivos hacia adelante (forward stepwise), basado en la probabilidad del estadístico de razón de verosimilitud (máxima verosimilitud). En la Tabla 4 indicamos los valores correspondientes a los tres análisis diferentes efectuados. Los valores corresponden al momento del último paso efectuado en los análisis, exceptuando los niveles de $0^{2}$ que reflejan las variaciones en los mismos a medida que se incluían en la ecuación nuevas variables significativas. El estadístico de Wald contrasta la hipótesis nula de que el coeficiente de regresión sea cero.
En un primer análisis, las cinco dimensiones (subescalas) de los miedos (puntuaciones de intensidad de miedo) constituyeron conjuntamente las variables independientes. Solamente resultó significativa la inclusión de la variable Miedos a pequeños animales y daños menores, la cual redujo significativamente el valor del $-2 \log$ de verosimilitud $\left(\chi^{2}=\right.$ $47,2, p<0,001$ ). Resulta interesante constatar que, a pesar de tratarse de una sola variable predictora (i.e., miedos a los animales), ésta resultaba eficaz para clasificar correctamente el $75,6 \%$ de los casos (el $83,3 \%$ de los niños y el $64,7 \%$ de las niñas) (véase la Tabla 4). Por tanto, los restantes tipos de miedos, entendidos como dimensiones generales, no parecían relevantes para discriminar entre ambos grupos (tras incluir en la ecuación la variable de miedos a los animales).

Cuando, empleando idéntico modelo de análisis de regresión logística, utilizamos las puntuaciones de prevalencia en lugar de los valores de intensidad, obtuvimos resultados muy semejantes $\left(\chi^{2}=\right.$ $43,0, p<0,001$; fueron clasificados correctamente el $71,2 \%$ de los casos). Tal

Tabla 4. Contenido de los miedos y diferenciación sexual (niñas vs. niños)

\begin{tabular}{|c|c|c|c|c|c|c|c|}
\hline Paso & Variable independiente & B & $x^{2}$ & Wald & $\mathrm{CC}(\%)$ & CP & OR (IC 95\%) \\
\hline 1 & $\begin{array}{l}\text { Subescalas FSSC-R (intensidad) } \\
\text { Miedos a pequeños animales }\end{array}$ & 0,29 & 47,2 & 30,7 & 75,6 & 0,42 & $8,1(2,8-22,8)$ \\
\hline $\begin{array}{l}1 \\
2\end{array}$ & $\begin{array}{l}\text { Subescalas FSSC-R (prevalencia) } \\
\text { Miedos a pequeños animales } \\
\text { Miedos al peligro y muerte }\end{array}$ & $\begin{array}{l}0,39 \\
0,14\end{array}$ & $\begin{array}{l}38,2 \\
43,0\end{array}$ & $\begin{array}{r}12,1 \\
4,6\end{array}$ & $\begin{array}{l}70,9 \\
70,9\end{array}$ & $\begin{array}{l}0,22 \\
0,12\end{array}$ & $\begin{array}{l}7,2(2,6-20,2) \\
4,1(1,4-11,5)\end{array}$ \\
\hline $\begin{array}{l}1 \\
2 \\
3 \\
4 \\
5\end{array}$ & $\begin{array}{l}\text { Items FSSC-R } \\
\text { Las lagartijas } \\
\text { Quedarse solo/a en casa } \\
\text { Las armas de fuego } \\
\text { Los murciélagos o los pájaros } \\
\text { Las películas de misterio }\end{array}$ & $\begin{array}{l}1,72 \\
0,88 \\
1,03 \\
1,46 \\
0,54\end{array}$ & $\begin{array}{l}53,3 \\
72,6 \\
83,6 \\
91,5 \\
98.2\end{array}$ & $\begin{array}{r}15,3 \\
5,4 \\
8,7 \\
6,2 \\
2,8\end{array}$ & $\begin{array}{l}79,6 \\
82,4 \\
82,9 \\
83,8 \\
83,9\end{array}$ & $\begin{array}{l}0,27 \\
0,14 \\
0,19 \\
0,14 \\
0,10\end{array}$ & $\begin{array}{r}14,4(3,2-64,3) \\
10,4(2,9-36,5) \\
4,6(2,3-9,1) \\
13,8(1,7-72,6) \\
7,3(3,2-16,8)\end{array}$ \\
\hline
\end{tabular}

Nota: Las variables independientes están separadas de acuerdo con su pertenencia a cada uno de los tres análisis de regresión logística efectuados.

Excepto para $\chi^{2}$, los valores corresponden al momento final del análisis (último paso).

Ambos estadísticos ( $\chi^{2}$ y Wald) son significativos estadísticamente $(p<0,001)$ en todos los casos indicados en la tabla.

$\mathrm{B}=$ Coeficiente de regresión logística

$\mathrm{CC}(\%)=$ Porcentaje de casos clasificados correctamente.

$\mathrm{CP}=$ Coeficiente de correlación parcial.

$\mathrm{OR}=$ odds ratio (tasa de posibilidad); entre paréntesis se indican los intervalos de confianza (IC) del $95 \%$. 
vez la diferencia más notable fue en que aquí resultó también discriminativa la variable Miedos a peligros físicos y muerte, si bien su capacidad predictora fue menor.

A modo exploratorio establecimos un nuevo análisis de regresión logística múltiple, tomando ahora como variables independientes los items del FSSC- $R$ (i.e., basándonos en las puntuaciones dadas a cada item), para de este modo determinar qué miedos concretos eran más eficaces para predecir la variable sexo, es decir, para conocer qué items diferenciaban mejor a los niños de las niñas. Tras emplear el método indicado de selección por pasos, solamente 5 variables independientes resultaron estadísticamente predictoras, esto es, los miedos relacionados con las lagartijas, las armas, quedarse sólo en casa, los murciélagos/pájaros, y las películas de misterio. En todas estas variables las niñas habían obtenido puntuaciones significativamente mayores que los niños $p<0,001)$. Los estadísticos correspondientes a este análisis de regresión logística están indicados en la Tabla 4.

Puesto que todos los tipos de miedos indicados en la Tabla 4 parecían capaces de discriminar entre los niños y las niñas, deseamos conocer hasta qué punto el sexo constituía un factor de riesgo para tales miedos. Para ello calculamos individualmente los niveles del estadístico odds ratio (OR; tasa de posibilidad) tras dicotomizar los valores de las variables de miedo indicadas. A las tres variables de miedo de las subescalas de intensidad y prevalencia se les asignó valor 1 cuando su valor superaba a la media en una desviación típica, y valor 0 en caso contrario. Así mismo, a las variables correspondientes a los 5 items del FSSC$R$ seleccionados (ver tabla) se les asignó valor 1 cuando su valor era de 3 (i.e., miedo excesivo), y valor 0 en caso de que tuviese valores inferiores (i.e., 1 o 2). En la tabla referimos los valores del estadístico OR, el cual compara la posibilidad de presentar un tipo de miedo cuando el sujeto es niña con la posibilidad de presentar dicho miedo cuando el sujeto es niño. Un OR de 1,0 indica que no existe relación entre ser niña y el tipo de miedo en cuestión (i.e., ambas variables serían independientes, no modificándose la ventaja o posibilidad). Un OR de 2,0 indicaría que el miedo ocurre el doble de veces entre las niñas que entre los niños. En la columna derecha de la Tabla 4 indicamos los valores de la OR, los cuales, aun siendo en general altos, han resultado particularmente elevados para los miedos relacionados con los pequeños animales (las lagartijas y los murciélagos o los pájaros). Se indican, así mismo, los intervalos confidenciales para el $95 \%$.

\section{DISCUSIÓN}

Los datos apoyan nuestra hipótesis general de que los miedos son fenómenos bastante comunes durante las edades infantiles, así como también la hipótesis de que las niñas suelen referir mayor número e intensidad de miedos que los niños. Estos resultados son bastante semejantes a los aportados por otros autores, en particular a los datos hallados por el grupo de Ollendick (1983; Ollendick et al., 1985, 1989, 1991). Nuestros resultados también son coherentes con los presentados por autores que han empleado versiones modificadas del FSSC-R (e.g., Gullone y King, 1993).

De acuerdo con nuestros datos, aunque la prevalencia de los miedos parece ser bastante elevada tanto en los niños como en las niñas, en éstas se evidencian niveles mucho mayores. Las niñas, como grupo, refieren experimentar una media de 24 miedos significativos (en los niños, la media de miedos excesivos es de 15). Estos niveles de prevalencia son sólo 
ligeramente mayores a los referidos por Ollendick et al. (1989) para muestras amplias de niños y adolescentes de Estados Unidos y Australia $(\bar{x}=18$ para varones y $\bar{x}=10$ para mujeres). No obstante, aparte de otras posibles razones que justifiquen estas diferencias, dichas medias corresponden a niños y adolescentes, mientras que las presentadas por nosotros (Tabla 1) se refieren a niños entre 9 y 11 años (como se ha sugerido, los miedos podrían declinar con el paso de la edad). De nuestro estudio parece desprenderse, pues, que los miedos excesivos son relativamente comunes durante la infancia, ya que la muestra refiere experimentar cerca de 20 miedos significativos.

$\mathrm{Al}$ estudiar cuáles eran los diez miedos más comunes (señalados por los niños/as como excesivos), observamos que todos correspondían a la dimensión de temores a peligros físicos y muerte, con la única excepción de un temor de tipo interpersonal (Item 15), el cual aparece también entre los diez más frecuentes (véase la Tabla 2). Observamos que más del $60 \%$ de los participantes estimaron poseer mucho miedo a estos estímulos o situaciones. Una vez más, pues, los datos hablan en favor de la elevada frecuencia con que ocurren los miedos en los niños. Si comparamos nuestros hallazgos con los referidos por Ollendick et al. (1989, 1991) observamos que entre ambos existe un gran paralelismo. Sólo dos de los miedos referidos por Ollendick et al. (1989) entre los diez más comunes no aparecen en nuestra Tabla 2; estos miedos correspondían a los Items 29 (obtener malas notas) y 11 (las serpientes). Nos llama la atención que los tres miedos más comunes en nuestro estudio sean también los tres más comunes en el estudio transcultural de Ollendick et al. (1989) y en el estudio con muestras británicas de Ollendick et al. (1991). También se repite la preponderancia de los miedos asociados al peligro y muerte.
Entre los once miedos que indicamos en la Tabla 2, solo tres no son señalados por Ollendick et al. (1989) dentro del grupo de los 10 temores más comunes. Estos son los miedos relacionados con recibir una descarga eléctrica, ser enviado al director del colegio, y los miedos a los microbios o padecer una enfermedad grave. Estos dos últimos temores, no obstante, sí aparecen entre los 10 más comunes en el estudio de Ollendick et al. (1991). Puesto que los datos de estos últimos autores fueron obtenidos con población de edades (entre 8 y 10 años) similares a las de nuestros participantes, parece razonable suponer que las leves discrepancias con los resultados de Ollendick et al. (1989) se deben más a diferencias de edad que a diferencias culturales. La preponderancia, entre los miedos más comunes, de los temores asociados a los peligros mayores y la muerte también ha sido referida recientemente por Gullone y King (1993).

En cuanto a las diferencias entre niños y niñas, podemos observar que ambos coinciden en 9 miedos entre los 10 más comunes (Tabla 3). El temor «Recibir una descarga eléctrica» ocupa el cuarto lugar entre los niños y en undécimo lugar entre el grupo de niñas. El temor "Que un ladrón entre en tu casa» es el cuarto miedo más común entre las niñas, pero sólo ocupa el duodécimo lugar en el grupo de niños. Aparte de estas pequeñas diferencias, puede observarse, sin embargo, que el patrón de los diez miedos más representativos es muy semejante en ambos grupos de participantes. En términos generales, nuestros resultados no difieren apenas de los presentados por Ollendick et al. (1989), ya que estos autores encontraron, así mismo, una coincidencia entre niños y niñas en 9 de los 10 miedos más frecuentes.

Tras analizar las diferencias entre niños y niñas en la intensidad de miedo, obtenemos resultados equivalentes a los 
constatados con muestras de otras culturas (Dong et al., 1994; Gullone y King, 1993: Ollendick et al., 1989, 1991). Es decir, las niñas suelen reflejar siempre niveles más elevados de miedo que los niños, tanto en la puntuación general de miedo como en las puntuaciones de las diferentes subescalas. En nuestro estudio, si bien las diferencias entre niños y niñas son significativas en todas las dimensiones de los miedos (véase la Tabla 3), merece la pena resaltar el hecho de que las diferencias parecen menos marcadas para la dimensión de miedos sociales (fracaso y crítica). Este resultado, de alguna forma, viene a corroborar otros hallazgos que sugieren la existencia de diferencias menores entre niños y niñas en relación con los miedos y ansiedad sociales, al menos si se comparan con las diferencias que se evidencian para otros tipos de miedos y fobias (véase Sandín, 1997).

Puesto que las niñas parecían puntuar siempre más alto en cada una de las diferentes dimensiones de los miedos, en principio todas estas categorias de miedos podrían resultar útiles para discriminar a los niños de las niñas, aunque posiblemente no todas tendrían el mismo poder discriminativo. Mediante los análisis de regresión logística analizamos hasta qué punto estas dimensiones resultaban eficaces para discriminar (o predecir) entre niños y niñas. En este sentido, constatamos que prácticamente sólo la categoría de miedos a los pequeños animales y daños menores parecía resultar significativa, la cual, por sí sola, era eficaz para clasificar correctamente al $75,6 \%$ de la muestra. La OR para esta variable resultó también relevante $(\mathrm{OR}=$ $8,1)$, lo que indica que las niñas tienen un riesgo ocho veces mayor de experimentar estos miedos que los niños.

Resultaba también necesario analizar el contenido de los miedos teniendo en cuenta cada uno de los items del cuestionario, aparte de las dimensiones genera- les de miedos. Sólo cinco miedos (items) parecían aportar una contribución única respecto a la predicción de la variable dependiente (i.e., sexo): las lagartijas, quedarse solo en casa, las armas de fuego, los murciélagos o los pájaros, y las películas de misterio. Debe tenerse en cuenta que, de forma aislada, muchos otros items también contribuyen significativamente a dicha predicción; sin embargo, en el análisis de regresión logística múltiple dichas variables dejan de ser relevantes, quedando como significativas únicamente las cinco señaladas (posiblemente porque muchos items de miedo comparten elevados niveles de varianza). Este modelo de regresión logística multivariada, por otra parte, parece bastante consistente y resultó eficaz para clasificar correctamente cerca del $84 \%$ de los participantes. Si observamos los valores estadísticos que presentamos en la Tabla 4, podemos constatar que los niveles más elevados de la OR corresponde a los miedos relativos a pequeños animales, i.e., a los miedos asociados a las lagartijas (OR $=14,4)$ y a los murciélagos y pájaros $(O R=13,8)$ (a pesar de que el item sobre el miedo a los murciélagos o pájaros no entró en la ecuación hasta el cuarto paso).

A modo de conclusión general, podemos afirmar que nuestro estudio aporta datos normativos y predictivos sobre los miedos infantiles basados en el cuestionario de autoinforme FSSC-R. Nuestros resultados apoyan los hallazgos obtenidos por otros autores desde diferentes culturas, y reafirman los postulados de que (1) los miedos son muy frecuentes durante las edades infantiles, (2) los miedos son más frecuentes e intensos en las niñas, y (3) los miedos relativos al peligro físico y la muerte poseen un especial relieve durante estas edades. Por otra parte, aportamos nuevos datos que sugieren a los temores asociados a los pequeños animales como los que mejor diferencia a las niñas de los niños. Finalmente, 
mediante el presente estudio se ofrecen datos relevantes en favor de la validación (datos normativos y validez predictiva o discriminante) de la versión española del cuestionario FSSC-R. Futuros trabajos, no obstante, deberían corroborar estos datos empleando muestras más amplias de niños normales, utilizando muestras con un espectro más amplio de edades, y empleando muestras de sujetos clínicos, en especial niños y adolescentes con trastornos de ansiedad.

\section{REFERENCIAS BIBLIOGRÁFICAS}

Dong, Q., Yang, B., y Ollendick, T.H. (1994).

Fears in Chinese children and adolescents and their relations to anxiety and depression. Journal of Child Psychology and Psychiatry, 35, 351-363.

Gullone, E. y King, N.J. (1992). Psychometric evaluation of a revised fear survey shedule for children and adolescents. Journal of Child Psychology and Psychiatry, 33, 987-998.

Gullone, E. y King, N.J. (1993). The fears of youth in the 1990's: Contemporary normative data. The Journal of Genetic Psycho$\log y, 154,137-153$.

King, N.J., Gullone, E., y Ollendick, T.H. (1990). Fears in children and adolescents with chronic mental conditions. Journal of Clinical Child Psychology, 19, 173-177.

McCanthie, H. y Spence, S.H. (1991). What is the revised Fear Survey Schedule for Children measuring? Behaviour Research and Therapy, 29, 495-502.

Ollendick, T.H. (1983). Reliability and validity of the Revised Fear Survey Schedule for Children (FSSC-R). Behaviour Research and Therapy, 21, 685-692.

Ollendick, T.H., King, N.J., y Frary, R.B. (1989). Fears in children and adolescents: Reliability and generalizability across gender, age and nationality. Behaviour Research and Therapy, 27, 19-26.

Ollendick, T.H., Matson, J.L., y Hensel, W.J. (1985). Fears in children and adolescents: Normative data. Behaviour Research and Therapy, 23, 465-467.

Ollendick, T.H., Yule, W., y Ollier, K. (1991). Fears in British children and their relationship to manifest anxiety and depression. Journal of Child Psychology and Psychiatry, 32, 321-331.

Pelechano, V. (1981). Miedos infantiles y terapia familiar-natural. Valencia: Alfaplus.

Pelechano, V. (1984). Programas de intervención psicológica en la infancia. Valencia: Alfaplús.

Sandín, B. (1995). Teorías sobre los trastornos de ansiedad. En A. Belloch, B. Sandín y F. Ramos (Eds.), Manual de psicopatología (Vol. 2, pp. 113-164). Madrid: McGrawHill.

Sandín, B. (1997). Ansiedad, miedos y fobias en niños y adolescentes. Madrid: Dykinson.

Sandín, B., y Chorot, P. (1995). Síndromes clínicos de la ansiedad. En A. Belloch, B. Sandín y F. Ramos (Eds.), Manual de psicopatología (Vol. 2, pp. 81-112). Madrid: McGraw-Hill.

Sandín, B., y Chorot, P. (1998). Dimensiones de los miedos en niños: Estructura factorial del FSSC-R. Psiquis, 19, 23-32.

Sherer, M.W. y Nakamura, C.Y. (1968). A fear survey chedule for children. Behaviour Research and Therapy, 6, 172-182. 